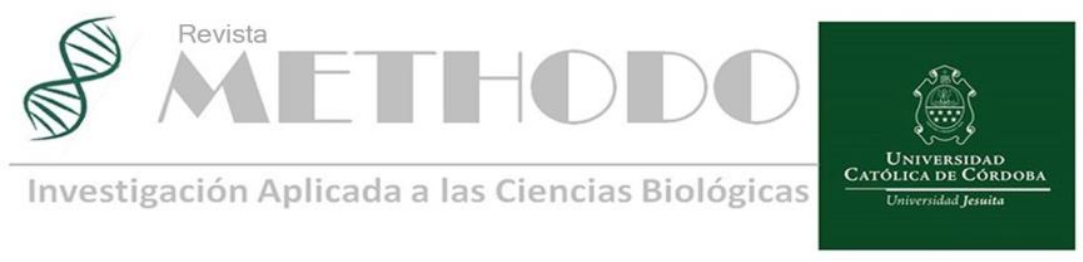

CASO CLINICO Rev. Methodo 2021;6(4):194-198 https://doi.org/10.22529/me.2021.6(4)08

Recibido 14 Jun. 20201| Aceptado 20 Jul. 2021|Publicado 05 Oct. 2021

\title{
Sialometaplasia necrotizante: presentación de un caso, dificultades de diagnóstico y revisión de la bibliografía
}

\section{Necrotizing sialometaplasia: presentation of a case, diagnostic difficulties and review of the bibliography}

\author{
Nicolás Leonardi ${ }^{1}$ (D) , Diego Sifuentes Cock $^{2}$, Gabriela Sambuelli³ ${ }^{3}$ Ignacio Gutiérrez Magaldi ${ }^{4}$, René \\ Luis Panico ${ }^{5}$ \\ 1. Universidad Católica de Córdoba, Facultad de Ciencias de la Salud, Cátedra Medicina Bucal \\ 2. Universidad Católica de Córdoba, Facultad de Ciencias de la Salud, Clínica Universitaria Reina Fabiola, Servicio de Otorrinolaringología \\ 3. Universidad Católica de Córdoba, Facultad de Ciencias de la Salud, Clínica Universitaria Reina Fabiola, Servicio de Anatomía Patológica \\ 4. Universidad Católica de Córdoba, Facultad de Ciencias de la Salud, Clínica Universitaria Reina Fabiola, Servicio de Medicina Interna \\ 5. Universidad Católica de Córdoba, Facultad de Ciencias de la Salud, Cátedra Medicina Bucal \\ Correspondencia: Nicolás Leonardi, e-mail: nico_leonardi@hotmail.com.ar
}

\section{Resumen}

La sialometaplasia necrotizante (SN) es una patología estadísticamente infrecuente, benigna, inflamatoria, autolimitante, que afecta predominantemente a las glándulas salivales menores. Su etiología no está clara, la mayoría de los autores sugieren que una lesión química, física o biológica de los vasos sanguíneos produciría cambios isquémicos, que desencadenaría infarto del tejido glandular con necrosis, inflamación e intento de reparación. Su aspecto clínico e histológico tiene apariencia de malignidad, pudiendo inducir a un diagnóstico erróneo de neoplasia maligna de origen anexial, considerando que la SN se trata de una patología autoresolutiva, es fundamental realizar un correcto diagnóstico clínico e histopatológico para evitar tratamientos quirúrgicos innecesarios. Se presenta el caso clínico de una paciente de sexo femenino que fue derivada al Servicio de Otorrinolaringología (ORL) de la Clínica Universitaria Reina Fabiola con diagnóstico de SN, sus características clínicas, histopatológicas y la evolución de la lesión.

Palabras clave: sialometaplasia necrotizante, ulceras orales, glándulas salivales

\begin{abstract}
Necrotizing sialometaplasia (NS) is a statistically infrequent disorder of benign, inflammatory and selflimiting nature that predominantly affects minor salivary glands. Its aetiology is not clear; most authors suggest that a chemical, physical or biological lesion of blood vessels would produce ischaemic changes leading to gland tissue stroke with necrosis, inflammation and healing attempt. Clinical and histological features of the lesion resemble malignancy and thus may lead to misdiagnose annexial malignant neoplasia, when in fact NS is self-healing. Therefore, an accurate clinical and histopathological diagnosis is essential to avoid mutilating or unnecessary surgical treatments. The present report describes the case of a female patient referred to the ENT department at Clínica Universitaria Reina Fabiola with NS diagnosis, its clinical and histopathological characteristics and evolution of the lesion.
\end{abstract}

Keywords: necrotizing sialometaplasia, oral ulcers, salivary glands

Revista Methodo: Investigación Aplicada a las Ciencias Biológicas. Universidad Católica de Córdoba Jacinto Ríos 571 Bo Gral. Paz. X5004FXS. Córdoba. Argentina. Tel.: (54) 3514517299 / Correo: methodo@ucc.edu.ar / Web: methodo.ucc.edu.ar | CASO CLINICO Rev. Methodo 2021;6(4):194-198. 


\section{Introducción}

La sialometaplasia necrotizante ( $\mathrm{SN}$ ) corresponde a un proceso inflamatorio, benigno y autoresolutivo. La localización característica es en zonas donde existan glándulas salivales menores, principalmente en el paladar duro o en el límite entre paladar duro y blando, siendo la afectación típicamente unilateral, aunque, de manera ocasional, puede presentarse en forma bilateral o en la línea media ${ }^{1-2-3}$. Sin embargo, es posible observarla también en la cara interna de los labios, piso de boca, lengua, trígono retromolar ${ }^{3-7}$, glándulas salivales mayores ${ }^{3-8-9}$, mucosa sinusal ${ }^{3-}$ ${ }^{10} \mathrm{y}$ en la laringe $\mathrm{e}^{3-11}$.

La etiología más aceptada es que una agresión físico-química o biológica sobre los vasos sanguíneos produciría isquemia, la cual conduciría al infarto del tejido glandular y su posterior necrosis ${ }^{3-12-13}$, inflamación e intento de reparación induciendo metaplasia, cambios en ductos $\mathrm{y}$ posterior cicatrización ${ }^{3-14}$. Algunos de los factores involucrados en esa alteración vascular serían: traumatismos locales directos, como los producidos por intubaciones, aplicación de anestesia local asociada a vasoconstrictores ${ }^{3-15-17}$, procedimientos quirúrgicos, uso de prótesis desadaptadas, vómitos provocados, como los descriptos en pacientes con bulimia ${ }^{18}$, radioterapia, uso de tabaco y cocaína entre otros serían factores involucrados en esta alteración vascular ${ }^{19-22}$. Algunos autores encuentran asociación con patologías como diabetes mellitus ${ }^{7} \mathrm{o}$ alcoholismo crónico $^{3-23-24}$.

Clínicamente la SN puede presentarse como una úlcera con bordes eritematosos, ligeramente elevados con un lecho necrótico de aspecto crateriforme, márgenes indurados y bien delimitados, ubicada comúnmente en paladar, pudiendo ser bilateral ${ }^{3-25}$, o bien como un aumento de volumen de consistencia fluctuante ${ }^{26}$, doloroso o asintomático ${ }^{27}$. Las características histopatológicas incluyen metaplasia escamosa de conductos y acinos, necrosis por coagulación de algunos lóbulos, pero conservando la arquitectura $\operatorname{lobular}^{28}$, presencia de tejido de granulación con infiltrado inflamatorio inespecífico e hiperplasia pseudoepiteliomatosa del epitelio mucoso superficial $^{3-29}$.

La SN es relativamente inusual, con una prevalencia del $1 \%$ del total de las biopsias ${ }^{2-25}$. El objetivo de nuestro trabajo es establecer los diagnósticos alternativos o diferenciales de esta entidad, en base a la clínica y a los hallazgos histopatológicos ya que, a pesar de ser un proceso poco frecuente, fácilmente puede confundirse con neoplasias malignas orales como linfomas, Carcinoma Adenoideo Quístico, Carcinoma
Mucoepidermoide o el Carcinoma de Células Escamosas ${ }^{12-16-20}$. Por lo tanto, es esencial arribar a un correcto y oportuno diagnóstico tanto clínico como histopatológico para evitar tratamientos quirúrgicos mutilantes e innecesarios.

\section{Caso clínico}

Se presenta una paciente de sexo femenino de 16 años que fue derivada desde el Servicio de Emergencias al Servicio de Otorrinolaringología de la Clínica Universitaria Reina Fabiola por intolerancia oral y odinofagia a causa de lesiones ulceradas en la cavidad bucal de 7 días de evolución.

La paciente refería la aparición de lesiones ulceradas y dolorosas inicialmente en la mucosa yugal del lado izquierdo 7 días previos a la consulta, la cual fue tratada con analgesia durante los primeros días con escasa respuesta al tratamiento empleado al momento de la consulta. Relata que durante esa semana permaneció afebril. Esto se acompañó en las últimas 48 horas de hiporexia debido a la odinofagia. A la inspección clínica se observaron múltiples lesiones ulceradas en la cavidad bucal afectando mucosa yugal del lado izquierdo, (Figura 1) y paladar blando (Figura 2), de consistencia ligeramente aumentada, esta última con un aspecto clínico crateriforme y la que ocasionaba mayor sintomatología. Las demás estructuras de la mucosa bucal y faríngea junto al examen otorrinolaringológico fueron normales. Debido a la intolerancia oral se decidió la internación, solicitud de estudios complementarios, además de interconsultas con los Servicios de Infectología, Reumatología, Dermatología y Clínica Médica. La serología para VDRL, VHS 1 y 2, VIH, VHC, VHB fueron negativas $y$ el hemograma indico valores compatibles con anemia microcítica hipocrómica con perfil de hierro orientado a anemia ferropénica, respetando las restantes series medulares por lo cual se descartó sospechar de un proceso linfoproliferativo en primera instancia.

A las 24 horas se controlaron las lesiones; debido al rápido aumento de tamaño y evolución de las mismas se decidió realizar interconsulta con un estomatólogo, donde se observó, una lesión ulcerada en el paladar blando, con fondo necrótico que comprometía hasta la úvula. Otra lesión en mucosa yugal izquierda, se observó estable y se podía ver la aparición de nuevas lesiones clínicas en la mucosa yugal contralateral (Figura 3) y en cara ventral de lengua (Figura 4).

Se decide realizar una biopsia de tipo incisional de la lesión de mucosa yugal derecha de reciente 
aparición, a las 48 horas se recibe el informe anatomopatológico arribando al diagnóstico compatible de Sialometaplasia Necrotizante en concordancia con la sospecha clínica.

En los cortes histológicos seriados se observaron acinos glandulares mucoserosos, correspondientes a glándulas salivales menores, con infiltrado inflamatorio mixto con áreas de necrosis (Figura 5), en relación a conductos glandulares con metaplasia escamosa que obstruían parcialmente la luz de los mismos (Figura 6). Estos hallazgos son compatibles con el diagnóstico de Sialometaplasia Necrotizante.

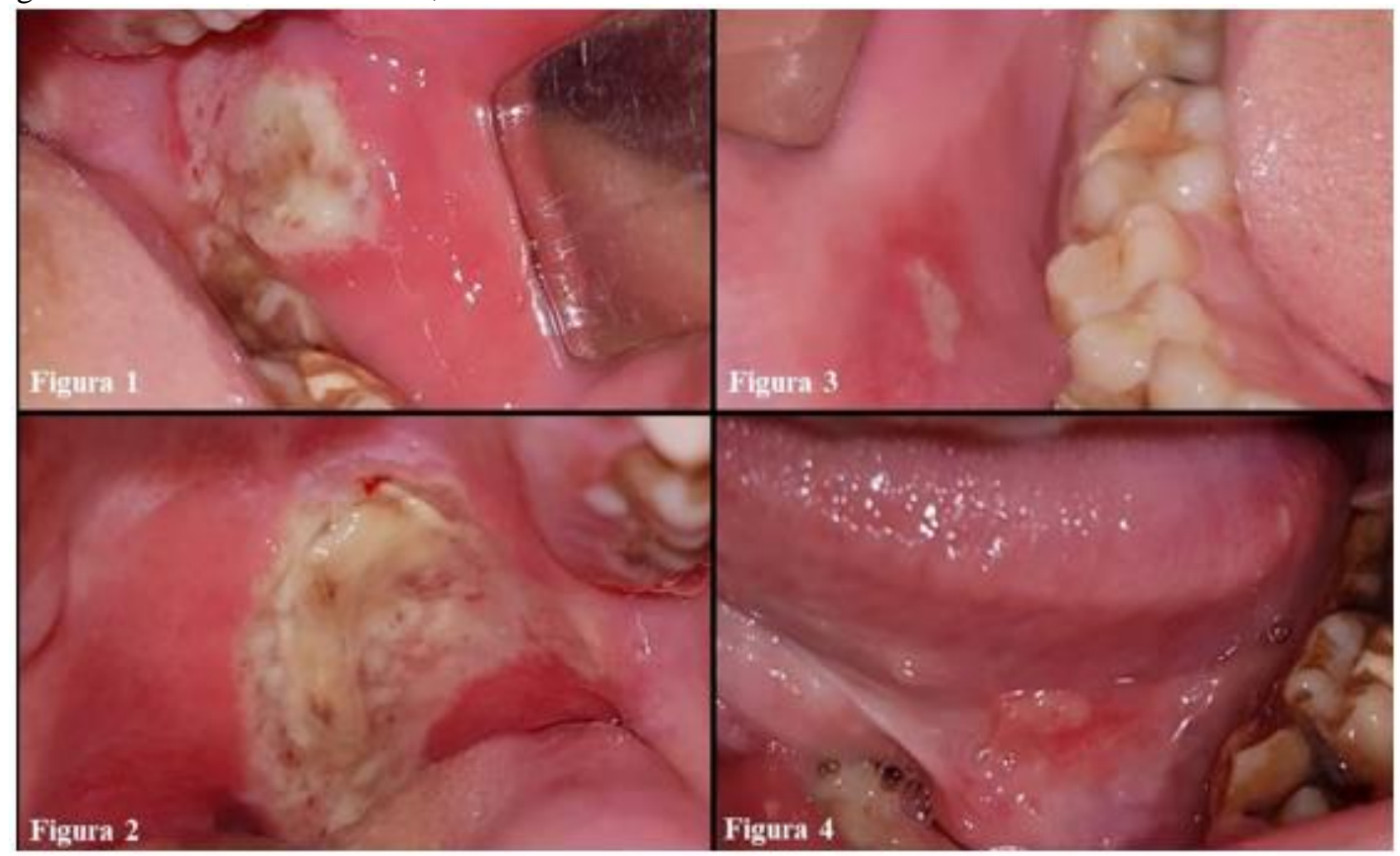

Figura 1. Lesión ulcerada ubicada en tercio posterior de mucosa yugal izquierda con un aspecto clínico necrótico. Figura 2. Lesión ulcerada ubicada en paladar blando con un aspecto clínico crateriforme y fondo necrótico comprometiendo hasta la úvula.

Figura 3. Lesión erosiva con un aspecto clínico necrótico en su superficie sobre una base eritematosa.

Figura 4. Lesión erosiva ubicada en cara ventral de lengua rodeada de un halo eritematoso.

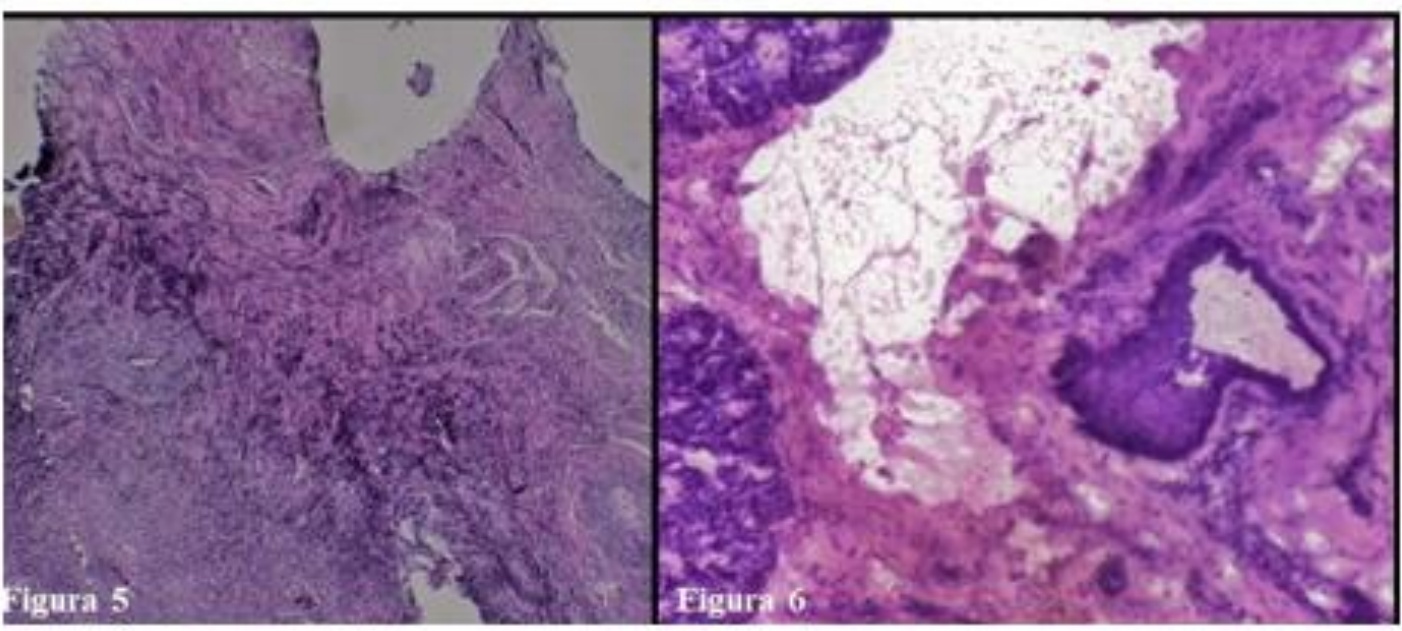

Figura 5 y 6. En los cortes histológicos seriados se observaron acinos glandulares mucoserosos, correspondientes a glándulas salivales menores, con infiltrado inflamatorio mixto y áreas de necrosis en relación a conductos glandulares con metaplasia escamosa que obstruían parcialmente la luz de los mismos. Estos hallazgos son compatibles con el diagnóstico de Sialometaplasia Necrotizante.

\section{Discusión}

La SN fue descripta por primera vez en 1973 por Abrams et al. como una lesión inflamatoria necrotizante de las glándulas salivales menores del paladar duro' ${ }^{1}$. Se trata de una afección relativamente rara, con una mayor prevalencia en mujeres de mediana edad ${ }^{2-30}$, cuya presentación clínica más común es la ulcerativa y que se

Revista Methodo: Investigación Aplicada a las Ciencias Biológicas. Universidad Católica de Córdoba. Jacinto Ríos 571 Bo Gral. Paz. X5004FXS. Córdoba. Argentina. Tel.: (54) 3514517299 / Correo: methodo@ucc.edu.ar / Web: methodo.ucc.edu.ar | CASO CLINICO Rev. Methodo 2021;6(4):194-198. 
presenta generalmente de forma unilateral, aunque existen reportes sobre SN bilaterales y en la línea media $^{31}$. En el caso anteriormente presentado, la relevancia del mismo radica en que las lesiones ulceradas se presentaron en una mujer joven, cuyas localizaciones fueron multifocales lo que lo hace aún más desafiante al diagnóstico en conjunto con la evolución clínica de la patología, afectando paladar blando, úvula, ambas mucosas yúgales y cara ventral de lengua.

Si bien, la etiología permanece desconocida, la mayoría de los autores aceptan la teoría de una interrupción de la irrigación sanguínea a una glándula salival menor como origen de la lesión y su relación con antecedentes de traumatismos o irritantes locales como el tabaco, consumo de cocaína, ácido gástrico en cuadros de reflujo gastroesofágico o bulimia ${ }^{13}$, alcohol, radiación, infecciones de la vía aérea superior, procedimientos quirúrgicos e infiltración de anestesia local en la zona ${ }^{17}$. Este último punto se describe con frecuencia en la literatura, sobre todo asociado al uso de vasoconstrictores en la mucosa palatina. En el caso presentado, otro aspecto importante es la aparición de las lesiones sin antecedentes previos encontrados en la bibliografía como factores desencadenantes de la SN.

Es fundamental una historia clínica minuciosa, además del trabajo interdisciplinario entre los profesionales de la salud para el diagnóstico clínico e histopatológico de certeza, debido al amplio abanico de patologías que pueden afectar a las glándulas salivales. Es importante también destacar que para el diagnóstico anatomopatológico la muestra obtenida mediante biopsia debe ser profunda, ya que la superficie de la lesión solo nos muestra el componente fibrinoleucocitario de la ulcera. Es de vital importancia realizar un correcto y oportuno diagnóstico a los efectos de evitar tratamientos quirúrgicos mutilantes e innecesarios, debido a que se trata de un proceso de resolución espontanea.

\section{Bibliografía}

1. Abrams AM, Melrose RJ, Howell FV. Necrotizing sialometaplasia. Cancer 1973;32:130-5.

2. Gahona Gutiérrez O; Beytía Erazo J; Pérez Lagos L; Argandona Pozo J. sialometaplasia necrotizante: reporte de un caso y revisión de la literatura. Rev Chil Cir. 2017;69(3):252--255

3. Femopase FL, Hernández SL, Gendelman H, Criscuolo MI, López de Blanc SA. Sialometaplasia Necrotizante: Presentación de cinco casos clínicos. Med Oral 2004;9:3048 .

4. Papanayotou PH, Kayavis JG, Epivatianos AA, Trigonidis G. Necrotizing sialometaplasia of the cheek: report of case and review of literature. J Oral Surg 1980;38:438-40.

5. Speechley JA, Anne Field E, Scott J. Necrotizing sialometaplasia occuring during pregnancy: Report of a case. J Oral Maxillofac Surg 1988;46:696-9.

6. Pulse CL, Levobics RS, Zegarelli DJ. Necrotizing sialometaplasia: report of a cas after lower lip mucocele excision. J Oral Maxillofac Surg 2000;58:1419-21.

7. Dominguez FV, Espinal EG. Sialometaplasie necrosante. Presentation de deux cas et revue de la litterature. Revue D odontostomatologie. 1982;11:375-8.

8. Rossie KM, Allen CM, Burns RA. Necrotizing sialometaplasia: A case with metachronous lesions. J Oral Maxillofac Surg 1986;44:1006

9. Russo A, Dell'Aquila A, Prota V, Sica GS. Scialometaplasia necrotizzantedell ghiandola sottomandibolare.Descrizione di un caso. Minerva Stomatol 1998;47:2737.

10. Hovinga J, Dejager H. A patient with necrotizing sialometaplasia. Int J Oral Surg 1977;6:280-2.

11. Grillon GL, Lally ET. Necrotizing sialometaplasia: literature review and presentation of five cases. J Oral Surg 1981;39:747-53.

12. Suckiel JM, Davis WH, Patakas BM, Kaminishi RM. Early and late manifestations of necrotizing sialometaplasia. J Oral Surg 1978;36:902-5.

13. Imai, T. \& Michizawa, M. Necrotizing sialometaplasia in a patient with an eating disorder: palatal ulcer accompanied by dental erosion due to binge-purging. J. Oral Maxillofac. Surg., 71(5):87985, 2013.

14. Chaudhry AP, Yamane GM, Salman L, Salman S, Saxon M, Pierri LK. Necrotizing sialometaplasia of palatal minor salivary glands: A report of 2 cases. $\mathrm{J}$ of Oral medicine 1985;40:2-4.

15. Shigematsu H, Shigematsu Y, Noguchi Y, Fujita K. Experimental study on necrotizing sialometaplasia of the palate in rats. Role of local anesthesic injections. Int Oral Maxillofac Surg 1996;25:239-41. 
16. De Saint Aubain Somerhausen N, Larsimont D, Tant L, Verhest A. Necrotizing sialometaplasia: apropos of a case and review of literature. Acta Stomatol Belg 1996;93:613.

17. Carlson, D. L. Necrotizing sialometaplasia: a practical approach to the diagnosis. Arch. Pathol. Lab. Med., 133(5):692-8, 2009.

18. Schoning H, Emshoff R, Kreczy A. Necrotizing sialometaplasia in two patients with bulimia and chronic vomiting. Int J Oral Maxillofac Surg 1998; 27:463-5.

19. Arguelles MT, Viloria JB, Talens MJ, Mc Crory TP. Necrotizing sialometaplasia. Oral Surg 1976;42:86-90.

20. Fechner RE. Necrotizing sialometaplasia. Am J Clin Pathol 1977;67:315-7.

21. Yoshimura Y, Matsuura R, Sugihara T, Matsumoto K. J Osaka Univ Dent Sch 1985;25:171-6.

22. Imbery TA, Edwards PA. Necrotizing sialometaplasia: literature review and case reports. J Am Dent Assoc 1996;127:1087-92.

23. Bannayan G, Fox G, Tilson HB. Necrotizing sialometaplasia of the palate. J Oral Surg 1976;34:727-30.

24. Murphy J, Giunta J, Meyer I, Robinson K. Necrotizing sialometaplasia. Oral Surg 1977; 44: 419-24.
25. Raugi, G. J. \& Kessler, S. Necrotizing sialometaplasia. A condition simulating malignancy. Arch. Dermatol., 115(3):329-31, 1979.

26. Santis, H. R.; Kabani, S. P.; Roderiques, A. \& Driscoll, J. M. Necrotizing sialometaplasia: an early, nonulcerative presentation. Oral Surg. Oral Med. Oral Pathol., 53(4):387-90, 1982.

27. Joshi, S. A.; Halli, R.; Koranne, V. \& Singh, S. Necrotizing sialometaplasia: A diagnostic dilemma! J. Oral Maxillofac. Pathol., 18(3):420-2, 2014

28. Favia G, Pratelli D, Maiorano E. La sialometaplasia necrotizzante. Analisi clinicopatologica di 6 casi. Minerva Stomatol 1996;45:121-7.

29. Williams, R. F. Necrotizing sialometaplasia after bronchoscopy. J. Oral Surg., 37(11):8168, 1979.

30. Rye, L. A.; Calhoun, N. R. \& Redman, R. S. Necrotizing sialometaplasia in a patient with Buerger's disease and Raynaud's phenomenon. Oral Surg. Oral Med. Oral Pathol., 49(3):233-6, 1980.

31. Birkholz, H.; Minton, G. A. \& Yuen, Y. L. Necrotizing sialometaplasia: review of the literature and report of nonulcerative case. J. Oral Surg., 37(8):588-92, 1979.

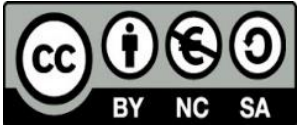

